\title{
ASSOCIAÇÃO DE HERBICIDAS PARA O MANEJO DE PLANTAS DANINHAS EM MILHO
}

\section{ASSOCIATION OF HERBICIDES FOR THE MANAGEMENT OF WEEDS IN CORN}

Adilson Lemos Rezende ${ }^{\mathrm{a}}$, Leandro Galon ${ }^{\mathrm{a} *}$, Bernardo Berenchtein ${ }^{\mathrm{b}}$, Cesar Tiago Forte ${ }^{\mathrm{c}}$, Emanuel Rodrigo de Oliveira Rossetto ${ }^{\mathrm{b}}$, Leonardo Brunetto ${ }^{\mathrm{a}}$,

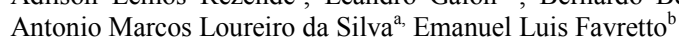

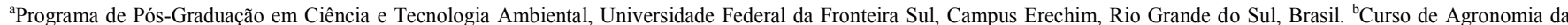
Universidade Federal da Fronteira Sul, Campus Erechim, Rio Grande do Sul, Brasil. ${ }^{\mathrm{c}}$ Promotor de Vendas Sênior, Diamaju Agrícola/Syngenta Crop, Rio Grande do Sul, Brasil.

*Autor correspondente: leandro.galon@uffs.edu.br.

\section{INFORMAÇÕES DO ARTIGO}

\section{Histórico do artigo:}

Recebido: 14 Dezembro 2020.

Aceito: 17 Março 2021.

Publicado: 31 Março 2021.

\section{Palavras-chave/Keywords:}

Digitaria ciliaris (Retz.) Koeler/ Digitaria ciliaris (Retz.) Koeler. Urochloa plantaginea (Link) Webster/ Urochloa plantaginea (Link) Webster.

Zea mays L./ Zea mays L..

Mistura em tanque/ Tank mix.

Financiamento:

CNPq, FAPERGS, UFFS e FINEp.

Direito Autoral: Este é um artigo de acesso aberto distribuído sob os termos da Licença Creative Commons, que permite uso, distribuição e reprodução irrestritos em qualquer meio, desde que $\mathrm{o}$ autor $\mathrm{e}$ a fonte originais sejam creditados.

\section{Citação deste artigo:}

REZENDE, A. L.; GALON, L.; BERENCHTEIN, B.; FORTE, C. T.; ROSSETTO, E. R. O.; BRUNETTO, L.; SILVA, A. M. L.; FAVRETTO, E. L. Associação de herbicidas para o manejo de plantas daninhas em milho. Revista Brasileira de Herbicidas, v. 19, n. 4. 2020.

\begin{abstract}
RESUMO
O uso de herbicidas para o controle de plantas daninhas na cultura do milho tem sido uma prática muito comum pelos produtores, sendo aplicados de modo isolado ou em mistura em tanque. Diante disso, o objetivo do trabalho foi avaliar a eficiência, a fitotoxicidade, o efeito em características fisiológicas e produtivas do milho após a aplicação de herbicidas de modo isolado ou em mistura em tanque. $\mathrm{O}$ experimento foi instalado a campo, no delineamento de blocos casualizados, com quatro repetições. Como tratamentos foram utilizados de forma isolada ou em mistura em tanque o glyphosate, [atrazine+simazine], nicosulfuron, mesotrione, tembotrione, mais uma testemunha capinada. Avaliou-se a fitotoxicidade dos herbicidas ao híbrido de milho AG 9025 PRO3, a eficiência no controle de papuã (Urochloa plantaginea) e milhã (Digitaria ciliaris) aos 7, 14, 21 e 28 dias após a aplicação dos tratamentos (DAT). Aos 50 DAT aferiu-se ainda as variáveis referentes às características fisiológicas do milho e na colheita determinou-se a produtividade de grãos. A fitotoxicidade ocasionada pelos herbicidas não foi perceptível ao híbrido de milho AG 9025 PRO3. O controle foi eficiente nos tratamentos que apresentaram a mistura em tanque de glyphosate com nicosulfuron e tembotrione. A taxa fotossintética aumentou com a aplicação de nicosulfuron e mesotrione ao se comparar com os demais tratamentos. A concentração interna de $\mathrm{CO}_{2}$, taxa de transpiração, condutância estomática de vapores de água, eficiência de carboxilação e uso eficiente da água não foram alteradas ao se usar os herbicidas isolados ou em mistura. A aplicação dos herbicidas não interferiu na produtividade de grãos de milho. A associação de glyphosate com outros herbicidas demonstra ser prática favorável para melhorar a eficácia de controle.
\end{abstract}

\section{ABSTRACT}

Corn is an important crop for agriculture, playing a fundamental role in animal and human feed and energy production. Therefore, the purpose of this study was evaluate the effectiveness, selectivity and effects on corn physiological and productive characteristics after herbicide application either isolated or in tank mix. The procedure adopted was randomized blocks design, with four replications each. The treatments, glyphosate, [atrazine + simazine], nicosulfuron, mesotrione, tembotrione, plus a weeded control were used alone or in a tank mixture. It was evaluated the herbicide phytotoxicity in AG 9025 PRO3 hybrid corn, as well as the weed control of alexander grass and crabgrass at 7, 14, 21 and 28 days after treatment (DAT). At 50 DAT, variables related to the grain physiological characteristics were also measured. Finally, during harvest, grain yield was determined. All evaluations showed that phytotoxicity caused by herbicides was not visually perceptible in AG 9025 PRO3 corn hybrid. The control was effective in treatments that presented in tank mix treatments glyphosate with nicosulfuron and tembotrione. The physiological characteristic affected after herbicide application was photosynthesis, which, when compared to other treatments, showed better results with nicosulfuron and mesotrione. Herbicides did not interfere in corn grain yield. Glyphosate associated with other herbicides used in corn crops shows to be a valuable practice to improve and accelerate the weed control studied with selectivity to the crop. 


\section{Introdução}

As culturas agrícolas, incluindo o milho, dependem de fatores de solo e de clima que podem influenciar no seu crescimento, desenvolvimento com consequências na produtividade e na qualidade do produto colhido (BASSO et al., 2018). Sabe-se que, além dos fatores edafoclimáticos, as plantas daninhas podem comprometer a produtividade das culturas e interferir no sistema de produção empregado (WANDSCHEER et al. 2014; BASSO et al., 2018).

Dentre as plantas daninhas que competem com o milho pelos recursos do ambiente destaca-se o Urochloa plantaginea (papuã/capim-marmelada) e a Digitaria ciliaris (milhã/capim-colchão) sendo que essas espécies estão presentes em várias regiões do Brasil, principalmente no Sul e no Alto Uruguai do Rio Grande do Sul (KISSMANN; GROTH, 1997; GALON et al., 2018a).

As plantas daninhas afetam negativamente a cultura do milho, reduzindo em mais de $30 \%$ a produtividade de grãos, como visto por Galon et al. (2018a) ao trabalharem com herbicidas aplicados isoladamente ou em mistura em tanque para o controle de U. plantaginea, Euphorbia heteroplylla (leiteiro) e Ipomoea indivisa (corda-de-viola). Os biótipos do gênero Digitaria também interferem na produtividade de grãos quando em competição com as culturas (DAN et al., 2010; BASSO et al., 2018), em especial ao milho, por pertencerem à mesma família botânica.

O manejo das plantas daninhas infestantes do milho é medida de controle que precisa ser tomada, sendo o método químico o mais utilizado em função da eficiência, rapidez e menor custo quando comparado a outros métodos de controle (TIMOSSI; FREITAS, 2011). Com o advento da tecnologia do milho resistente ao herbicida glyphosate (Roundup Ready ${ }^{\circledR}$ ), que visa aumentar o espectro de controle, teve-se novas alternativas de associação de herbicidas na cultura, com isso melhorando a eficiência sobre diversas plantas daninhas (CHIKOYE; LUM; UDENSI 2010).

Porém, é importante salientar que o uso dessa tecnologia mudará as populações de plantas daninhas para aquelas que têm resistência ao glyphosate, caso o uso de somente um herbicida seja posicionado na cultura (OPEÑA et al., 2014). Já existem casos de resistência do gênero Digitaria aos herbicidas inibidores de acetolactato sintase (ALS), dos gêneros Digitaria e Urochloa aos inibidores de FS II e alguns casos de resistência de outras espécies de plantas daninhas (Conyza spp., Lolium multiflorum, Amaranthus spp.) aos inibidores da 5-enolpiruvilshikimate-3 -fosfato sintase (EPSPs), ALS e da Acetil-Co A carboxilase (ACCase) (AGOSTINETTO; VARGAS, 2014; HEAP, 2020).

Assim, é possível destacar a importância de manejos com associações de herbicidas de diferentes mecanismos de ação para diminuir o surgimento de novos casos de plantas daninhas resistentes ou mesmo o controle das tolerantes, em especial ao glyphosate, no mundo e no Brasil. As associações de herbicidas, além de demonstrarem melhora no controle de plantas daninhas necessitam apresentarem seletividade as culturas (CARVALHO; MORETTI; SOUZA, 2010; GALON et al., 2018a). Os herbicidas como atrazine, nicosulfuron, mesotrione e tembotrione quando aplicados associados em pós-emergência, apresentam-se como alternativa para o controle de U. plantaginea, I. indivisa e E. heterophylla mantendo o milho livre da infestação (WILLIAMS et al., 2010; GALON et al., 2018a), pelo menos no período crítico de controle, quando se diferenciam os componentes de rendimento da cultura (GALON et al., 2018b).

É de fundamental importância a rotação de herbicidas e de mecanismos de ação no manejo de plantas daninhas infestantes das culturas agrícolas, pois proporciona um maior espectro de controle de espécies, diminui o banco de sementes do solo, reduz a probabilidade do surgimento de biótipos resistentes e pode garantir bons resultados de produtividade (OPEÑA et al., 2014; FORTE et al., 2018; WESTWOOD et al., 2018).

Além do estudo sobre o controle de plantas daninhas que os herbicidas ocasionam, torna-se importante estabelecer os prejuízos que esses produtos podem causar nos parâmetros fisiológicos das plantas de milho como na atividade fotossintética, transpiração foliar, concentração interna de $\mathrm{CO}_{2}$, condutância estomática, uso eficiente da água e eficiência da carboxilação, sejam eles aplicados de modo isolado ou associados (GALON et al., 2020).

Dessa forma, estabelecer alternativas para maior espectro de controle de plantas daninhas sem prejudicar a produtividade da cultura do milho é essencial para a sustentabilidade do sistema de manejo. Portanto, a hipótese desse trabalho é de que a associação de herbicidas em tanque proporciona melhor controle de plantas daninhas com menor fitotoxicidade ao milho, porém com possíveis alterações nas variáveis fisiológicas e de produtividade de grãos. Diante disso, objetivou-se com o trabalho avaliar a eficiência, a fitotoxicidade e o efeito em características fisiológicas e produtivas do milho após a aplicação de herbicidas de modo isolado ou em mistura em tanque.

\section{Material e Métodos}

O experimento foi conduzido em campo na área experimental da Universidade Federal da Fronteira Sul (UFFS), Campus Erechim, RS, de outubro de 2018 a abril de 2019. A semeadura do milho foi efetuada em sistema de plantio direto na palha, sendo que 30 dias antes dessa operação efetuou-se a dessecação da vegetação com o herbicida glyphosate $+2,4-\mathrm{D}\left(1440+1209 \mathrm{~g} \mathrm{ha}^{-1}\right)$ para que a cultura emergisse livre da infestação de plantas daninhas. Na Figura 1 é ilustrada as condições meteorológicas no decorrer da condução do experimento em campo, obtidas no Inmet (2020). 


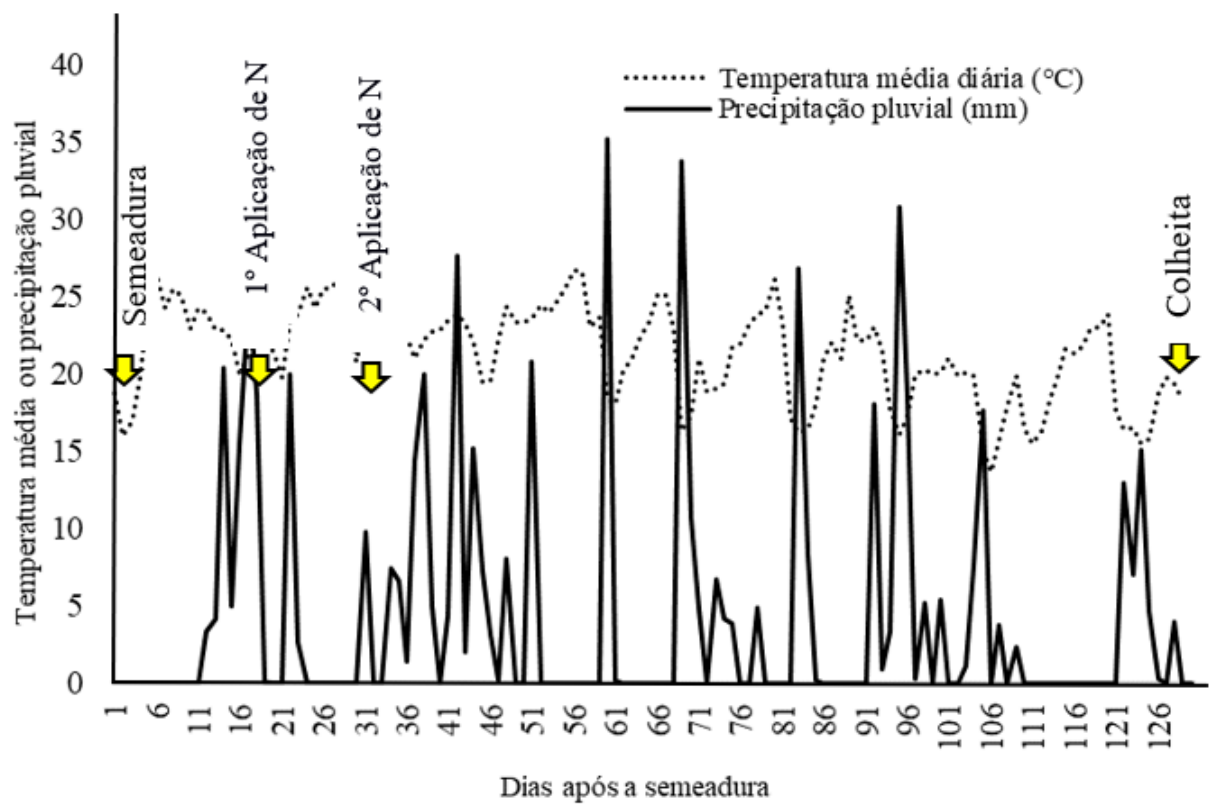

Figura 1. Temperatura média do ar e precipitação pluviométrica diária no período de condução do experimento, outubro de 2018 a abril de 2019.

O experimento foi instalado em delineamento de bloco casualizados com quatro repetições. Os tratamentos utilizados no experimento estão descritos na Tabela 1, juntamente com as doses e momento da aplicação. Cada unidade experimental foi caracterizada por uma parcela de
$15 \mathrm{~m}^{2}$ (5 x $\left.3 \mathrm{~m}\right)$, semeadas com o híbrido de milho AG 9025 PRO3 destinado à produção de silagem ou grãos, em 6 linhas no espaçamento de $0,5 \mathrm{~m}$ na densidade de 3,5 plantas $\mathrm{m}^{-1}$.

Tabela 1. Tratamentos utilizados no experimento, respectivas doses e época de aplicação para controle de plantas daninhas infestantes do híbrido de milho AG 9025 PRO3.

\begin{tabular}{|c|c|c|c|c|}
\hline Tratamentos & $\begin{array}{c}\text { Doses } \\
\left(\mathrm{g} \text { i.a. } \mathrm{ha}^{-1}\right)\end{array}$ & $\begin{array}{l}\text { Produto Comercial } \\
\text { Formulação }\end{array}$ & $\begin{array}{c}\text { Doses } \\
\left(\mathrm{L} / \mathrm{kg} \mathrm{h}^{-1}\right)\end{array}$ & $\begin{array}{c}\text { Adjuvantes } \\
(\% \mathrm{v} / \mathrm{v})\end{array}$ \\
\hline Testemunha capinada & --- & --- & & --- \\
\hline Glyphosate & 1335,0 & Roundup Original DI-SL ${ }^{1}$ & 3,0 & --- \\
\hline [Atrazine+simazine] & 1500,0 & Primatop- $\mathrm{SC}^{2}$ & 6,0 & Nimbus $(0,5)$ \\
\hline Mesotrione & 192,0 & Callisto-SC $\mathrm{SC}^{3}$ & 0,4 & Nimbus $(0,5)$ \\
\hline Nicosulfuron & 60,0 & Accent-WG ${ }^{4}$ & 0,045 & Aureo $(0,1)$ \\
\hline Tembotrione & 100,8 & Soberan-SC ${ }^{5}$ & 0,24 & Aureo $(0,5)$ \\
\hline Glyphosate + [atrazine + simazine $]$ & $1335,0+1500,0$ & Roundup Original + Primatop & $3,0+6,0$ & Nimbus $(0,5)$ \\
\hline Glyphosate + mesotrione & $1335,0+192,0$ & Roundup Original+Callisto & $3,0+0,4$ & Nimbus $(0,5)$ \\
\hline Glyphosate + nicosulfuron & $1335,0+60,0$ & Roundup Original+Accent & $3,0+0,045$ & Aureo $(0,1)$ \\
\hline Glyphosate + tembotrione & $1335,0+100,8$ & Roundup Original+Soberan & $3,0+0,24$ & Aureo $(0,5)$ \\
\hline
\end{tabular}

Empresas Fabricantes dos herbicidas: ${ }^{1}$ Monsanto do BrasilLtda, ${ }^{2,3}$ Syngenta Proteção de Cultivos Ltda, ${ }^{4}$ Du Pont do Brasil S.A e ${ }^{5}$ Bayer S.A.

A correção da fertilidade do solo, classificado como Latossolo vermelho aluminoférrico típico (EMBRAPA, 2013) foi realizada com base na análise química do mesmo, com as seguintes características: $\mathrm{pH}$ (água) $=5,1$; matéria orgânica $=3,0 \%$; argila $=>60 \% ; \mathrm{P}=5,2 \mathrm{mg} \mathrm{dm}^{-3}, \mathrm{~K}=118,0$ $\mathrm{mg} \mathrm{dm}{ }^{-3}, \mathrm{Ca}^{+2}=5,5$ cmolc dm ${ }^{-3} ; \mathrm{Mg}^{+2}=3,0 \mathrm{cmolc} \mathrm{dm}^{-3}$; $\mathrm{Al}^{+3}=0,3$ cmolc $\mathrm{dm}^{-3} ; \mathrm{H}+\mathrm{Al}=7,7 \mathrm{cmolc} \mathrm{dm}^{-3} ; \mathrm{CTC}$ efetiva $=16,6 \mathrm{cmolc} \mathrm{dm}^{-3}$.
Para efeitos de correção da fertilidade do solo seguiuse as recomendações de adubação para a cultura do milho (ROLAS, 2016). A adubação química no sulco de semeadura foi de $500 \mathrm{~kg} \mathrm{ha}^{-1}$ da fórmula 05-30-15 de N-P-K e aplicação de nitrogênio em cobertura, na forma de ureia, realizada em dois momentos, no estádio V4 e V8 (quatro e oito folhas completamente expandidas, respectivamente) da cultura, na dose de $90 \mathrm{~kg} \mathrm{ha}^{-1}$ de $\mathrm{N}$ em cada estádio. 
A aplicação dos herbicidas foi efetuada com pulverizador costal de precisão, pressurizado a $\mathrm{CO}_{2}$, equipado com quatro pontas de pulverização tipo leque DG 110.02, mantendo-se pressão constante de $210 \mathrm{kPa}$ e velocidade de deslocamento de $3,6 \mathrm{~km} \mathrm{~h}^{-1}$, o que proporcionou a vazão de $150 \mathrm{~L} \mathrm{ha}^{-1}$ de calda de herbicida. As condições ambientais no momento da aplicação dos herbicidas eram: céu nublado (50\%), temperatura média do ar de $24,1^{\circ} \mathrm{C}$, umidade relativa do ar de $63,4 \%$, solo úmido e ventos de 2 a $4 \mathrm{~km} \mathrm{~h}^{-1}$. A cultura estava no estádio de 5 a 6 folhas completamente desenvolvidas (V5 a V6) e as plantas daninhas de 2 folhas a 1 perfilho no momento da aplicação dos tratamentos em pós-emergência.

As avaliações de controle do papuã (U. plantaginea) e da milhã (D. ciliaris) foram efetuadas aos 7, 14, 21, 28 dias após a aplicação dos tratamentos (DAT). Aos 7, 14, 21 e 28 DAT foi realizado as avaliações de fitotoxicidade dos herbicidas ao milho. Foram atribuídas notas percentuais para as avaliações de controle ou de fitotoxicidade, onde zero $(0 \%)$ significa ausência de controle ou de fitotoxicidade às plantas daninhas ou a cultura e cem $(100 \%)$ representa a morte das espécies (VELINI; OSIPE; GAZZIERO, 1995). Pelo levantamento botânico constatou-se na área densidades médias das plantas daninhas de 132 e 67 plantas $\mathrm{m}^{-2}$ de $U$. plantaginea e de $D$. ciliaris, respectivamente.

As variáveis fisiológicas do milho foram avaliadas aos 50 DAT, sendo elas: a taxa fotossintética

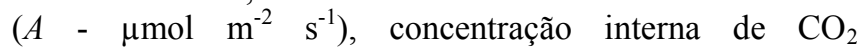
$\left(C i-\mu \mathrm{mol} \mathrm{mol}^{-1}\right)$, taxa de transpiração $\left(E-\mathrm{mol} \mathrm{H}_{2} \mathrm{O} \mathrm{m}^{-2} \mathrm{~s}^{-1}\right)$, condutância estomática de vapores de água

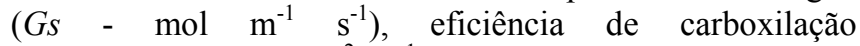
$\left(E C\right.$ - mol $\left.\mathrm{CO}_{2} \mathrm{~m}^{-2} \mathrm{~s}^{-1}\right)$ e uso eficiente da água
( $E U A$ - mol $\mathrm{CO}_{2} \mathrm{~mol} \mathrm{H}_{2} \mathrm{O}^{-1}$ ) aferidas no terço médio da última folha completamente expandida das plantas. A eficiência da carboxilação $\left(E C-\operatorname{mol} \mathrm{CO}_{2} \mathrm{~m}^{-2} \mathrm{~s}^{-1}\right)$ e a eficiência do uso da água ( $\left.E U A-\mathrm{mol} \mathrm{CO}_{2} \mathrm{~mol} \mathrm{H}_{2} \mathrm{O}^{-1}\right)$ foram calculadas a partir da razão das variáveis $A / C i$ e $A / E$, respectivamente. Para isso, utilizou-se um analisador de gases no infravermelho (IRGA), marca ADC, modelo LCA PRO (Analytical Development Co. Ltd, Hoddesdon, UK). Cada bloco foi avaliado sob iluminação natural em um dia, entre oito e dez horas da manhã, em condições de céu limpo, de forma que se mantivessem as condições ambientais homogêneas durante as análises.

Para a determinação da produtividade de grãos do milho foi realizado a trilha das espigas coletadas em área de $3 \mathrm{~m}^{-2}$ em cada unidade experimental e posteriormente foram aferidos o peso e a umidade, sendo essa corrigida para $13 \%$ e o peso extrapolado para $\mathrm{kg} \mathrm{ha}^{-1}$.

Os dados foram submetidos à análise de variância pelo teste $\mathrm{F}$, em sendo significativos as médias foram submetidas ao teste de Scott-Knott. Todos os dados foram analisados a $5 \%$ de probabilidade, utilizando-se o programa computacional Winstat (MACHADO; CONCENÇÃO, 2002).

\section{Resultados e Discussão}

Houve efeito significativo dos tratamentos testados para o controle de papuã (U. plantaginea), milhã ( $D$. ciliaris), para atividade fotossintética - $A$, eficiência de carboxilação - EC e eficiência de uso da água - EUA (Tabelas 2, 3 e 4$)$.

Tabela 2. Controle (\%) de papuã (Urochloa plantaginea) infestante do híbrido de milho AG 9025 PRO3 em função da aplicação de herbicidas de modo isolado ou associados ao glyphosate em pós-emergência inicial.

\begin{tabular}{lccccc}
\hline \multirow{2}{*}{ Tratamentos } & Dose & \multicolumn{4}{c}{ Controle de papuã (\%) } \\
\cline { 3 - 6 }$\left(\mathrm{g} \mathrm{i.a.} \mathrm{ha}^{-1}\right)$ & ---- & $7 \mathrm{DAT}^{1}$ & $14 \mathrm{DAT}$ & $21 \mathrm{DAT}$ & $28 \mathrm{DAT}$ \\
\hline Testemunha capinada & 1335,0 & $100 \mathrm{a}^{2}$ & $100 \mathrm{a}$ & $100 \mathrm{a}$ & $100 \mathrm{a}$ \\
Glyphosate & 1500,0 & $96 \mathrm{~b}$ & $97 \mathrm{a}$ & $98 \mathrm{a}$ & $98 \mathrm{a}$ \\
[Atrazine+simazine] & 192,0 & $26 \mathrm{f}$ & $58 \mathrm{~b}$ & $78 \mathrm{~b}$ & $85 \mathrm{~b}$ \\
Mesotrione & 60,0 & $54 \mathrm{e}$ & $53 \mathrm{~b}$ & $75 \mathrm{~b}$ & $80 \mathrm{~b}$ \\
Nicosulfuron & 100,8 & $69 \mathrm{~d}$ & $98 \mathrm{a}$ & $99 \mathrm{a}$ & $99 \mathrm{a}$ \\
Tembotrione & $1335,0+1500,0$ & $95 \mathrm{~d}$ & $98 \mathrm{a}$ & $100 \mathrm{a}$ & $99 \mathrm{a}$ \\
Glyphosate+[atrazine+simazine] & $1335,0+192,0$ & $98 \mathrm{~b}$ & $99 \mathrm{a}$ & $100 \mathrm{a}$ & $99 \mathrm{a}$ \\
Glyphosate + mesotrione & $1335,0+60,0$ & $97 \mathrm{~b}$ & $100 \mathrm{a}$ & $100 \mathrm{a}$ & $100 \mathrm{a}$ \\
Glyphosate + nicosulfuron & $1335,0+100,8$ & $98 \mathrm{~b}$ & $99 \mathrm{a}$ & $99 \mathrm{a}$ & $99 \mathrm{a}$ \\
Glyphosate + tembotrione & & 4,50 & 9,13 & 6,06 & 4,46 \\
\hline C.V. $(\%)$ & & &
\end{tabular}

${ }^{1}$ Dias após a aplicação dos tratamentos. ${ }^{2}$ Médias seguidas de mesmas letras minúsculas na coluna não diferem entre si pelo teste de Scott-Knott a $\mathrm{p}<0,05$.

O glyphosate aplicado isolado ou associado a [atrazine+simazine], mesotrione, nicosulfuron e tembotrione foram os tratamentos que apresentaram os melhores controles de $U$. plantaginea e $D$. ciliaris, dos 7 aos 28 DAT (Tabelas 2 e 3). Esse fato está ligado a mistura de herbicidas com mecanismos de ação distintos que aumenta o espectro de controle das plantas daninhas, ocorrendo assim efeito sinérgico, ou seja, maior resposta que quando aplicados de maneira isolada (GALON et al., 2010; BASSO et al., 2018; GALON et al., 2018a). É importante ressaltar que os herbicidas com rápida eficiência de controle, minimizam a competição inicial das plantas daninhas com a cultura do 
milho. Essa prática é importante para ser aliada a outros métodos de controle, como por exemplo, o manejo cultural, com uso de coberturas de solo, que em muitos casos pode reduzir para níveis de $15 \%$ a infestação de plantas daninhas, como por exemplo, Setaria italica e Chenopodium album (MHLANGA et al., 2016; LOWRY; BRAINARD, 2019; BÜCHI et al., 2019).

Tabela 3. Controle (\%) de milhã (Digitaria ciliaris) no híbrido de milho AG 9025 PRO3 em função da aplicação de herbicidas em isolado ou associados ao glyphosate em pós-emergência.

\begin{tabular}{lccccc}
\hline \multirow{2}{*}{ Tratamentos } & \multirow{2}{c}{$\begin{array}{c}\text { Dose } \\
\left(\mathrm{g} \mathrm{i.a.} \mathrm{ha}^{-1}\right)\end{array}$} & \multicolumn{4}{c}{ Controle de milhã (\%) } \\
\cline { 3 - 6 } & --- & $100 \mathrm{a}^{2}$ & $14 \mathrm{DAT}$ & $21 \mathrm{DAT}$ & $28 \mathrm{DAT}$ \\
\hline Testemunha capinada & 1335,0 & $96 \mathrm{~b}$ & $98 \mathrm{a}$ & $100 \mathrm{a}$ & $100 \mathrm{a}$ \\
Glyphosate & 1500,0 & $26 \mathrm{f}$ & $60 \mathrm{~b}$ & $85 \mathrm{~b}$ & $97 \mathrm{a}$ \\
[Atrazine+simazine] & 192,0 & $54 \mathrm{e}$ & $61 \mathrm{~b}$ & $83 \mathrm{~b}$ & $77 \mathrm{~b}$ \\
Mesotrione & 60,0 & $68 \mathrm{~d}$ & $98 \mathrm{a}$ & $99 \mathrm{a}$ & $99 \mathrm{a}$ \\
Nicosulfuron & 100,8 & $63 \mathrm{~d}$ & $98 \mathrm{a}$ & $99 \mathrm{a}$ & $99 \mathrm{a}$ \\
Tembotrione & $1335,0+1500,0$ & $88 \mathrm{c}$ & $99 \mathrm{a}$ & $100 \mathrm{a}$ & $100 \mathrm{a}$ \\
Glyphosate + [atrazine+simazine] & $1335,0+192,0$ & $98 \mathrm{~b}$ & $99 \mathrm{a}$ & $100 \mathrm{a}$ & $99 \mathrm{a}$ \\
Glyphosate + mesotrione & $1335,0+60,0$ & $97 \mathrm{~b}$ & $100 \mathrm{a}$ & $100 \mathrm{a}$ & $100 \mathrm{a}$ \\
Glyphosate + nicosulfuron & $1335,0+100,8$ & $98 \mathrm{~b}$ & $100 \mathrm{a}$ & $100 \mathrm{a}$ & $99 \mathrm{a}$ \\
Glyphosate + tembotrione & & 4,26 & 8,35 & 5,33 & 6,23 \\
\hline C.V. (\%) & & &
\end{tabular}

${ }^{1}$ Dias após a aplicação dos tratamentos. ${ }^{2}$ Médias seguidas de mesmas letras minúsculas na coluna não diferem entre si pelo teste de Scott-Knott a $\mathrm{p}<0,05$.

Tabela 4. Respostas fisiológicas expressas em concentração interna de $\mathrm{CO}_{2}\left(\mathrm{Ci}-\mu \mathrm{mol} \cdot \mathrm{mol}^{-1}\right)$, taxa transpiratória $\left(\mathrm{E}-\mathrm{mol}_{2} \mathrm{O} \mathrm{m}^{-2} \mathrm{~s}^{-1}\right)$, condutância estomática de vapores de água $\left(\mathrm{Gs}-\mu \mathrm{mol} \cdot \mathrm{mol}^{-1}\right)$, taxa fotossintética $\left(\mathrm{A}-\mu \mathrm{mol} \mathrm{m} \mathrm{m}^{-2} \mathrm{~s}^{-1}\right)$, eficiência na carboxilação (EC - mol $\left.\mathrm{CO}_{2} \mathrm{~m}^{-2} \mathrm{~s}^{-1}\right)$ e eficiência no uso da água das plantas $\left(\right.$ EUA - mol $\mathrm{CO}_{2} \mathrm{~mol} \mathrm{H}_{2} \mathrm{O}^{-1}$ ) produtividade de grãos de milho - PROD $\left(\mathrm{kg}\right.$ ha $\left.{ }^{-1}\right)$ aos 50 dias após a aplicação dos tratamentos e em função da a aplicação de herbicidas de modo isolados ou em associação em tanque.

\begin{tabular}{lccccccc}
\hline \multicolumn{1}{c}{ Tratamentos } & $\mathrm{Ci}$ & $\mathrm{E}$ & $\mathrm{Gs}$ & $\mathrm{A}$ & $\mathrm{EC}$ & EUA & PROD \\
\hline Testemunha capinada & $235^{\text {ns }}$ & $1,15^{\mathrm{ns}}$ & $0,05^{\mathrm{ns}}$ & $4,84 \mathrm{~b}^{1}$ & $0,02 \mathrm{~b}$ & $4,20 \mathrm{~b}$ & $7055^{\mathrm{ns}}$ \\
Glyphosate & 256 & 1,28 & 0,06 & $5,16 \mathrm{~b}$ & $0,02 \mathrm{~b}$ & $4,34 \mathrm{~b}$ & 6597 \\
[Atrazine+simazine] & 230 & 1,30 & 0,06 & $3,65 \mathrm{~b}$ & $0,02 \mathrm{~b}$ & $2,91 \mathrm{~b}$ & 6837 \\
Mesotrione & 223 & 1,70 & 0,07 & $10,54 \mathrm{a}$ & $0,06 \mathrm{a}$ & $6,52 \mathrm{a}$ & 6641 \\
Nicosulfuron & 209 & 1,67 & 0,08 & $10,83 \mathrm{a}$ & $0,05 \mathrm{a}$ & $6,54 \mathrm{a}$ & 6608 \\
Tembotrione & 242 & 1,03 & 0,06 & $5,36 \mathrm{~b}$ & $0,02 \mathrm{~b}$ & $5,23 \mathrm{a}$ & 7347 \\
Glyphosate+[atrazine+simazine] & 228 & 1,35 & 0,06 & $6,71 \mathrm{~b}$ & $0,04 \mathrm{a}$ & $5,04 \mathrm{a}$ & 6996 \\
Glyphosate + mesotrione & 244 & 1,51 & 0,05 & $3,50 \mathrm{~b}$ & $0,02 \mathrm{~b}$ & $3,12 \mathrm{~b}$ & 6926 \\
Glyphosate + nicosulfuron & 214 & 1,50 & 0,08 & $6,26 \mathrm{~b}$ & $0,05 \mathrm{a}$ & $4,91 \mathrm{a}$ & 6226 \\
Glyphosate + tembotrione & 229 & 1,20 & 0,07 & $7,37 \mathrm{~b}$ & $0,04 \mathrm{a}$ & $5,74 \mathrm{a}$ & 6335 \\
\hline C.V.(\%) & 27,43 & 37,12 & 31,73 & 29,88 & 42,52 & 30,33 & 21,28 \\
\hline
\end{tabular}

${ }^{n s}$ não significativo a $p<0,05 .{ }^{1}$ Médias seguidas de mesmas letras minúsculas na coluna não diferem entre si pelo teste de ScottKnott a $\mathrm{p}<0,05$.

O uso de nicosulfuron e tembotrione ocasionou controle igual estatisticamente a testemunha capinada, dos 14 aos 28 DAT (Tabelas 2 e 3). Esses herbicidas testados isoladamente apresentaram controle satisfatório, não só de $U$. plantaginea e D. ciliaris, como também de corda-deviola (Ipomoea indivisa) e leiteiro (Euphorbia heterophylla) (GALON et al., 2018a; BASSO et al., 2018).

A aplicação do glyphosate para controle de $D$. ciliaris e U. plantaginea em pós-inicial proporcionou maior eficácia em mistura com os demais herbicidas, equivalendose a testemunha capinada (Tabelas 2 e 3). Todas as misturas com glyphosate, a partir dos 14 DAT apresentaram controle superior a 99\% para ambas as plantas daninhas avaliadas. Salienta-se que, para ser considerado eficiente, um herbicida precisa apresentar controle de determinada planta daninha superior a 80\% (OLIVEIRA; FREITAS; VIEIRA 2009).

Embora o controle de plantas daninhas em milho resistente ao glyphosate possa ser muito eficaz, o uso dessa tecnologia continuamente e sem rotação de mecanismos de ação poderá selecionar plantas daninhas resistentes ou tolerantes, e essas irão dominar o ambiente com o passar dos anos de cultivo (OPEÑA et al., 2014). Diante do exposto, se faz necessário a rotação de mecanismos de ação e a associação dos mesmos para melhorar o controle e se ter menor risco da ocorrência de resistência das plantas daninhas aos herbicidas (WESTWOOD et al., 2018).

Em relação a fitotoxicidade não foi observado efeito significativo dos tratamentos, demonstrando que tanto os 
herbicidas aplicados de modo isolado ou associados foram seletivos para o híbrido de milho AG 9025 PRO3 (dados não apresentados). Desse modo constata-se haver segurança em aplicar os tratamentos em isolado ou em associação com o glyphosate para o manejo de plantas daninhas no híbrido AG 9025 PRO3. Mesmo com a aplicação de altas doses de glyphosate, a degradação enzimática na cultura resistente ocorre sem impedimento, com elevado índice de substrato (NANDULA et al., 2019). Galon et al. (2018a), ao estudarem os herbicidas, atrazine+simazine, nicosulfuron, tembotrione e mesotrione, também presentes nesse estudo observaram baixa fitotoxicidade a cultura, sem prejudicar os componentes de rendimento e na produtividade de grãos dos híbridos de milho fórmula TL ${ }^{\circledR}$ e SYN 7B28.

Para as respostas fisiológicas da cultura do milho pode-se observar na Tabela 4, que a concentração interna de $\mathrm{CO}_{2}(\mathrm{Ci})$, a taxa de transpiração $(E)$ e a condutância estomática $(G s)$ não apresentam diferenças significativas com a aplicação dos herbicidas. Infere-se que essas variáveis são pouco influenciadas pela aplicação dos herbicidas testados. A provável causa disso pode estar relacionada com a ausência de fitotoxicidade que os tratamentos herbicidas ocasionaram ao hibrido de milho AG 9025 PRO3, conforme já relatado anteriormente. Torres et al. (2012) ao estudarem o efeito dos herbicidas, tembotrione, MSMA, diuron + hexazinone, sulfentrazone, trifloxysulfuron-sodium, tebuthiuron e clomazone, também observaram baixa influência em características fisiológicas das cultivares de cana-de-açúcar (RB867515, RB855156 e SP80-1816). De modo similar a aplicação de fomesafen+fluazifop-p-butyl em feijão não alterou as variáveis relacionadas a fisiologia da cultura (MANABE et al., 2014).

As maiores taxas fotossintéticas $(A)$ foram observadas com aplicação de mesotrione e nicosulfuron, sendo inclusive superiores que a testemunha capinada (Tabela 4). Mesmo sem apresentar fitotoxicidade para esses herbicidas, possivelmente houve maior demanda energética para a metabolização desses produtos na cultura do milho. Destaca-se que quando as plantas sofrem alguma injúria elas começam a desencadear mecanismos de defesa e normalmente ocorre um maior investimento na produção de energia, no caso a fotossíntese para conseguir metabolizar o herbicida, além da emissão de folhas novas livres dos sintomas dos produtos (Torres et al., 2012). Os demais tratamentos demonstraram a $A$ menores que o observado ao mesotrione e ao nicosulfuron, porém igualaram-se a testemunha capinada, não ocorrendo assim influências dos mesmos sobre o metabolismo do milho.

A eficiência de carboxilação $(E C)$ e do uso da água $(E U A)$ indicam que os herbicidas mesotrione, nicosulfuron $\mathrm{e}$ as misturas de [atrazine+simazine], nicosulfuron $\mathrm{e}$ tembotrione com o glyphosate foram os tratamentos que apresentaram as melhores performances (Tabela 4). Em relação aos demais tratamentos observou-se que foram iguais estatisticamente a testemunha capinada. Galon et al. (2020) também encontraram diferenças na $E C$ e no $E U A$ ao aplicarem sobre o híbrido de milho, SYN 488 VIP 3, glyphosate e saflufenacil de modo isolado ou associados, o que contribui em partes com os resultados do presente estudo.
Mesmo apresentando diferença no controle das plantas daninhas e com alterações nas variáveis fisiológicas $A, E C$ e $E U A$, a produtividade de grãos de milho não foi alterada pelo uso dos tratamentos (Tabela 4). É importante ressaltar que o melhor tratamento é aquele que alia rotação dos mecanismos de ação, eficiência no controle, evita o reabastecimento do banco de sementes de plantas daninhas do solo e não diminui a produtividade de grãos de milho (FORTE et al., 2018; GALON et al., 2018a). O uso de herbicidas na cultura do milho demonstrou aumento da produtividade de grãos de 1,5 a 8,5 vezes ao se comparar o uso de produtos contra o não uso (OPEÑA et al., 2014). Mesmo que estatisticamente todos os tratamentos não tenham diferido entre si, observou-se que o uso de tembotrione demonstrou 4 e $9,6 \%$ maior produtividade do que a testemunha capinada e a média dos demais tratamentos herbicidas utilizados, respectivamente.

Diante dos resultados fica evidente que os herbicidas nicosulfuron e tembotrione se destacaram em relação aos demais no controle das espécies de plantas daninhas bem como na seletividade e sem danos ao metabolismo fisiológico das plantas de milho.

\section{Conclusões}

Os herbicidas aplicados isoladamente e em mistura com glyphosate não causaram fitotoxicidade ao híbrido de milho AG9025 PRO3.

As características físiológicas do milho são pouco afetadas com a aplicação dos herbicidas isolados ou em associação com o glyphosate.

A taxa fotossintética aumentou com a aplicação de nicosulfuron e mesotrione ao se comparar com os demais tratamentos.

A associação de herbicidas com glyphosate aumenta a eficiência de controle de papuã (U. plantaginea) e milhã (D. ciliaris), sem alterar a produtividade de grãos de milho.

\section{Agradecimentos}

Ao CNPq, à FAPERGS, a UFFS e ao FINEP pelo auxílio financeiro à pesquisa e pelas concessões de bolsas.

\section{Referências}

Agostinetto, D.; Vargas, L. Resistência de plantas daninhas a herbicidas. Pelotas/RS: UFPel, 2014. 398 p.

Basso, F. J. M.; Galon, L.; Forte, C. T.; Agazzi, L. R.; Nonemacher, F.; Perin, G. F. Weed management in $R^{\circledR}{ }^{\circledR}$ maize with herbicides applied isolated or associated with glyphosate. Revista Ciência Agroveterinária, v. 17, n. 2, p. 148-157, 2018.

Büchi, L.; Wendling, M.; Amossé, C.; Jeangros, B.; Charles, R. Cover crops to secure weed control strategies in a maize 
crop with reduced tillage. Field Crops Research, v. 247, 107583, p .1-7, 2019.

Carvalho, F. T.; Moretti, T. B.; Souza, P. A. Eficácia e seletividade de associações de herbicidas utilizados em pósemergência na cultura do milho. Revista Brasileira de Herbicida, v. 9, n. 2, p. 35-41, 2010.

Chikoye, D.; Lum, A. F.; Udensi, E. U. Efficacy of a new glyphosate formulation for weed control in maize in southwest Nigeria. Crop Protection, v. 29, n. 9, p. 947-952, 2010 .

Dan, H. A.; Barroso, A. L.; Dan, L. G. M.; Finotto, T. R.; Feldkircher, C.; Santos, V. S. Controle de plantas daninhas na cultura do milho por meio de herbicidas aplicados em pré -emergência. Pesquisa Agropecuária Tropical, v. 40, n. 4, p. $388-393,2010$.

Embrapa - Empresa brasileira de pesquisa agropecuária. Sistema brasileiro de classificação de solos. Embrapa Solos. 2013; 154 p.

Forte, C. T.; Galon, L.; Beutler, A. N.; Basso, F. J.; Reichert Jr., F. W.; Perin, G. F.; Tironi, S. P. Soil management systems and their effect on the weed seed bank. Pesquisa Agropecuária Brasileira, v. 53, n. 4, p. 435-442, 2018.

Galon, L.; Tironi, S. P.; Ferreira, E. A.; Aspiazú, I.; Pinto, J. J. O. Avaliação do método químico de controle de papuã (Brachiaria plantaginea) sobre a produtividade do milho. Pesquisa Agropecuária Tropical, v. 40, n. 4, p. 414-421, 2010

Galon, L.; David, F. A.; Forte, C. T.; Reichert Jr., F. W.; Radunz, A. L.; Kujawinski, R. Chemical management of $\mathrm{s}$ in corn hybrids. Weed Biology and Management, v. 18, n. 1, p. 26-40, 2018a.

Galon, L.; Bagnara, M. A. M.; Gabiatti, R. L.; Reichert Jr., F. W.; Basso, F. J. M.; Nonemacher, F. Interference periods of weeds infesting maize crop. Journal of Agricultural Science, v. 10, n. 10, p. 197-205, 2018 b.

Galon, L.; Weirich, S. N.; Franceschetti, M. B.; Aspiazu, I.; Silva, A. F. da; Forte, C. T. Selectivity of saflufenacil applied alone or mixed to glyphosate in maize. Journal of Agricultural Studies, v. 8, n. 3, p. 775-787, 2020.

Heap, I. Weed Science (International Survey Of Herbicide Resistant Weeds). Disponível em: <http:// www.weedscience.org/Summary/Country.aspx?>. Acesso em: 15 fev. 2020

Inmet - Instituto Nacional de Meteorologia. Disponível em $<\mathrm{http}$ ://www.inmet.gov.br/portal/index.php?r=tempo2/ mapasPrecipitacao>. Acesso em: 10 dez. 2020.

Kissmann, K. G.; Groth, D. Plantas infestantes e nocivas. 2. ed. São Paulo: BASF. Tomo I. 1997, 825 p.
Lowry, C. J.; Brainard, D. C. Strip intercropping of ryevetch mixtures: Effects on weed growth and competition in strip-tilled sweet corn. Weed Science, v. 67, n. 1, p. 114125,2019

Machado, A. A.; Concenção, A. R. Sistema de análises estatísticas para Windows - WINSTAT (Versão 2.11). Pelotas: Núcleo de Informação Aplicada - Universidade Federal de Pelotas, 2002.

Manabe, P. M. S.; Matos, C. C.; Ferreira, E. A.; Silva, A. A.; Sediyama, T.; Manaba, A. Physiological characteristics of beans in competition with weed. Bioscience Journal, v. 30, n. 6 , p. 1721-1728, 2014.

Mhlanga, B.; Chauhan, B. S.; Thierfelder, C. Weed management in maize using crop competition: A review. Crop Protection, v. 88, n. 1, p. 28-36, 2016.

Nandula, V. K.; Riechers, D. E.; Ferhatoglu, Y.; Barret, M.; Duke, S. O.; Dayan, F. E. Herbicide Metabolism: Crop Selectivity, Bioactivation, Weed Resistance, and Regulation. Weed Science, v. 67, n. 2, p. 149-175, 2019.

Oliveira, A. R.; Freitas, S. P.; Vieira, H. D. Controle de Commelina benghalensis, C. erecta, Tripogandra diuretica na cultura do café. Planta Daninha, v. 27, n. 4, p. 823-830, 2009.

Opeña, J. L.; Quilty, J. R.; Correa Jr., T.; Chauhan, B. S. Weed population dynamics, herbicide efficacies, and crop performace in a sprinkler-irrigated maize-rice cropping system. Field Crops Research, v. 167, n. 2, p. 119-130, 2014.

Rolas - Rede oficial de laboratórios de análise de solo e de tecido vegetal. Manual de adubação e calagem para os estados do Rio Grande do Sul e Santa Catarina. Porto Alegre: Sociedade Brasileira de Ciência do Solo, 11, 2016. $376 \mathrm{p}$.

Timossi, P. C.; Freitas, T. T. Eficácia de nicosulfuron isolado e associado com atrazine no manejo de plantas daninhas em milho. Revista Brasileira de Herbicidas, v. 10, n. 3, p. 210-218, 2011.

Torres, L. G.; Ferreira, E. A.; Rocha, P. R. R.; Faria, A. T.; Gonçalves, V. A.; Galon, L. Changes in the physiological characteristics of sugarcane cultivars submitted to herbicide application. Planta Daninha, v. 30, n. 3, p. 581-587, 2012.

Velini, E. D.; Osipe, R.; Gazziero, D. L. P. Procedimentos para instalação, avaliação e analise de experimentos com herbicidas. Londrina: SBCPD, 1995. 42 p.

Wandscheer, A. C. D.; Rizzardi, M. A.; Reichert, M.; Gaviraghi, F. Capacidade competitiva da cultura do milho em relação ao capim-sudão. Revista Brasileira de Milho e Sorgo, v. 13, p. 129-141, 2014. 
A. L. REZENDE et al.

Westwood, J. H.; Charudattan, R.; Duke, S. O.; Fennimore, S. A.; Marrone, P.; Slaughter, D. C.; Swanton, C.; Zollinger, R. Weed Management in 2050: Perspectives on the future of weed science. Weed Science, v. 66, n. 3, p. 275-285, 2018.

Williams, M. M.; Boerboom, C. M.; Rabaey, T. L. Significance of atrazine in sweet corn weed management systems. Weed Technology, v. 24, n. 2, p. 139-142, 2010. 